\title{
Influencias y prescriptores del consumo teatral. Un estudio sobre el efecto de las críticas teatrales y otras instancias de prescripción en los hábitos de asistencia al teatro
}

\author{
$\mathrm{M}^{\mathrm{a}}$ Teresa Logroño TORMO \\ Universitat de València \\ malotor@alumni.uv.es
}

Recibido: 2 de febrero de 2015

Aceptado: 15 de septiembre de 2015

\begin{abstract}
Resumen
Este artículo analiza la influencia que ejercen diversas instancias de prescripción en los hábitos de asistencia al teatro. El estudio se basa en una encuesta realizada específicamente para esta investigación en la que fueron entrevistadas 210 personas que asistían a una representación teatral en tres salas distintas de la ciudad de Valencia. El estudio ha revelado la importancia del boca a boca en la decisión de asistir al teatro y su mayor influencia entre el público menos asiduo a las representaciones teatrales. Los resultados obtenidos también han puesto de manifiesto la existencia de una estrecha relación entre el efecto prescriptor de las críticas teatrales y las pautas de asistencia al teatro, así como su mayor influencia entre los espectadores de salas de orientación comercial y dirigidas al gran público.

Palabras clave: Cultura, artes escénicas, prescripción, criticas, sociología de la cultura.

\section{Influences and advice of theatrical consumption. A study about the effect of the theatre critics and other instances of evaluation in the habits of theatre attendance}

\begin{abstract}
This article analyses the influence that different criticism stages of proceedings exert in the habits of theatre attendance. The study is based on the survey carried out specifically for this research in which 210 people, who attended a theatrical representation, were interviewed in three different theatres in the city of Valencia. The study has revealed the mouth to mouth importance in the decision of attending the theatre and its stronger influence on the audiences who less frequently go to theatrical representations. The results obtained have also made clear the existence of a narrow relation between the advice effect of the theatre critics and the patterns of attendance to the theatre, just like its bigger influence between theatres with commercial orientation and those which are addressed to the broad audiences.
\end{abstract}

Keywords: Culture, performing arts, prescription, critics, sociology of culture.

\section{Referencia normalizada}

LOGROÑO TORMO, Ma Teresa (2016): "Influencias y prescriptores del consumo teatral. Un estudio sobre el efecto de las críticas teatrales y otras instancias de prescripción en los hábitos de asistencia al teatro". Estudios sobre el Mensaje Periodístico. Vol. 22, Núm. 1 (enero-junio), págs.: 391-410. Madrid, Ediciones Complutense.

Sumario: 1. Introducción. 2. Marco teórico. 3. Método; 3.1. Muestra; 3.2. Cuestionario; 3.3. Análisis de datos. 4. Resultados. 5. Conclusiones. 6. Referencias bibliográficas. 7. Anexo. Tablas de resultados.

\section{Introducción}

Sin público, uno de los componentes fundamentales del teatro, no existiría la experiencia escénica. Pero tan difícil como concebir una obra sin espectadores es descifrar su comportamiento. Los motivos, las preferencias y las actitudes de los consumido- 
res reales y potenciales obedecen a múltiples factores. La investigación que se presenta en este artículo analiza la influencia de los medios de comunicación -a través de las críticas teatrales-como estimuladores o inhibidores de la asistencia al teatro. ¿En qué medida condiciona leer una crítica? ¿Aumenta el público de un espectáculo teatral el hecho de que sea recomendado desde los medios de comunicación? ¿Cómo de importante es para las compañías y entidades culturales tener presencia en las secciones de cultura de los medios de comunicación?

El trabajo de investigación que se presenta parte del supuesto de que uno de los factores para incrementar la asistencia de espectadores al teatro es la disponibilidad de información relacionada con las artes escénicas (Colbert y Cuadrado, 2003; Kolb, 2005; Kotler y Scheff, 1997). Partiendo de esta premisa, se han planteado diversas hipótesis a través de las cuales se afirma que las piezas informativas publicadas en los medios de comunicación pueden influir en las pautas de asistencia y favorecer que el público potencial empiece a interesarse o aumente su interés por el teatro a partir del efecto de personalidades o líderes de opinión, sobre todo si éstos aparecen en medios de comunicación masivos (Cuadrado y Berenguer, 2002: 50). De acuerdo con estos antecedentes, el principal objetivo de este trabajo consiste en efectuar una aproximación empírica a los diversos prescriptores que influyen en la decisión de adquirir una entrada para asistir a una representación teatral con la finalidad de identificar qué variables -relacionadas con la información teatral- tienen una mayor influencia en tal decisión. A partir de este planteamiento se examina el comportamiento de los espectadores teatrales y el significado de sus tomas de decisión en un espacio como el de la información publicada en los medios de comunicación, en el que las entidades e instituciones culturales no pueden ejercer más que un mínimo control.

Con el fin de obtener datos empíricos se realizó un estudio del público teatral en la ciudad de Valencia en el que se examinaron sus hábitos teatrales, el grado de acceso y uso de los distintos canales de información cultural y el conocimiento y la influencia de las opiniones vertidas a través de las críticas teatrales y otras vías de prescripción presentes en los mass media. El trabajo se ha estructurado en cuatro grandes apartados. El primero presenta una revisión de los principales estudios sobre las pautas y factores sociales que inciden en la asistencia al teatro y constituye el marco teórico de la investigación. A continuación se incluye un apartado dedicado al planteamiento metodológico, en el cual se exponen las hipótesis y el diseño de la investigación, así como las características de la muestra y el procedimiento de análisis de datos. Posteriormente se plantean los resultados de la investigación y, por último, se presenta un apartado con las principales conclusiones.

\section{Marco teórico}

El Plan General del Teatro (en adelante, PGT) -aprobado en el año 2007, y revisado en 2011, por las distintas asociaciones empresariales y profesionales más representativas del sector (Instituto Nacional de las Artes Escénicas y la Cultura, 2011: 5)-, establecía como una de sus prioridades el desarrollo de públicos. Entre sus principales líneas estratégicas, el PGT señalaba que para promover la presencia social y el desarrollo de la demanda se debía "fomentar la presencia social del teatro a través de los 
medios de comunicación y las redes sociales, incrementando su presencia en los medios de comunicación, tanto públicos como privados, así como en Internet y en las redes sociales". Y es que durante los últimos años, la escasa presencia de la actividad teatral en los medios de comunicación no ha favorecido el interés social por el teatro. Los profesionales del teatro entrevistados en el estudio de Sánchez de Horcajo (1999), concordaban en afirmar que los medios de comunicación conceden poca relevancia al teatro e influyen débilmente en la afluencia de espectadores. Es por ello que el PGT señalaba que el sector teatral debía desarrollar acciones de promoción e implantar estrategias y técnicas de marketing cultural para responder de un modo más profesional a las necesidades de promoción y captación de públicos. El marketing, y en concreto las acciones de difusión a través de los medios de comunicación, son las herramientas de las que más necesitado se encuentra el teatro, habida cuenta de la conveniencia de identificar públicos potenciales para neutralizar las barreras que a menudo impiden su consumo. Los mass media pueden ejercer una notable influencia sobre los espectadores e incrementar el valor social de las artes escénicas, lo que puede llevar "al incremento de la demanda y permitirá que una parte del no público acceda por primera vez a una experiencia escénica y rompa el tabú del aburrimiento, el esfuerzo y el tedio que se asocia a la práctica cultural" (Sellas y Colomer, 2009: 22).

Desde este punto de vista, en el Foro Internacional de las Artes Escénicas celebrado en Bilbao, Colomer y Garrido (2010) ya planteaban entre sus retos la necesidad de implantar un sistema de información, prescripción y acompañamiento que resultara adecuado a los diversos públicos potenciales. Partiendo de la premisa de que además de disponer de una oferta accesible es necesario asegurar una adecuada comunicación de la misma, las principales conclusiones del foro destacaron la necesidad de una función prescriptiva que ayude a los públicos a tomar una elección adecuada. En este sentido, la comunicación cultural puede desarrollar una función orientadora y guiar al público hacia determinadas propuestas culturales a la hora de elegir entre distintas opciones (Izquierdo, 2014) y, más en concreto, la crítica periodística puede colaborar en la creación y consolidación de audiencias ya que "juega un papel relevante a la hora de disolver el lazo que cierne el dispositivo comunicativo destinado a proporcionar públicos masivos a la lógica del espectáculo y al mismo tiempo de cooperar en la experiencia del espectador competente, dispuesto a establecer un diálogo crítico con las más diversas prácticas escénicas" (Lorente, 2013: 1044). De esta manera, Fernández Torres había definido el papel del crítico teatral como "garante e inspector de la calidad del servicio teatral" capaz de advertir a los espectadores "de los engaños que le amenazan y del riesgo de que le vendan como teatro productos que no merecen el nombre de tal" (Fernández Torres, 1995: 52).

Para una parte de los espectadores, el estímulo que en ocasiones propicia la decisión de adquirir una localidad para asistir a una representación escénica es simplemente el anuncio atractivo de un espectáculo interesante. El cartel y quizás los actores propician esa decisión. Kotler y Scheff (1997: 149) mencionan un estudio llevado a cabo por el Theatre Royal Stratford East de Inglaterra y publicado diez años antes en la revista Artlink en el que se afirma que los espectadores asisten al teatro porque desean ver una obra o un actor determinados. Para ese tipo de personas, la asistencia a 
una representación escénica es a menudo "algo rutinario y habitual, y la decisión de compra es un proceso relativamente simple y de baja implicación". Por contra, hay otros perfiles de espectadores para los cuales el elemento clave a la hora de tomar la decisión de asistencia no es la obra en cartel, sino otros factores que forman parte de la experiencia global de asistir al teatro. En este sentido, la mayoría de factores que afectan a la decisión de asistencia, interés y nivel de satisfacción con una representación teatral van más allá de los aspectos específicos de la oferta escénica. Factores tan genéricos como las tendencias macroambientales y tan específicos como la propia psicología de cada individuo tienen una influencia esencial en la actitud de las personas respecto a la decisión que van a tomar, el dinero que se van a gastar, las experiencias que persiguen y las necesidades que tratan de cubrir (Cuadrado y Berenguer, 2002; Kolb, 2005; Kotler y Scheff, 1997). Es fundamental, por tanto, tener en cuenta que el proceso de toma de decisiones empieza mucho antes de la adquisición de una entrada. Este proceso, conocido como EKB por ser las iniciales de los autores que lo definieron (Engel, Kollat y Blackwell) a finales de los sesenta (Engel, Blackwell y Miniard, 1993) comprende cinco fases: reconocimiento de la necesidad, búsqueda de información, evaluación de las alternativas, decisión de compra y actitud después de la compra. La investigación que se presenta se sitúa en la segunda etapa del modelo $\mathrm{EKB}$, es decir, en la fase de búsqueda de información. Es en esa etapa cuando el espectador se muestra interesado por acceder a información adicional, realiza búsquedas activas en los medios de comunicación o Internet, consulta horarios y precios y comprueba la disponibilidad de entradas (Kolb, 2005: 101). Las fuentes de información de los consumidores pueden ser personales, como familia y amigos; comerciales, publicidad y carteles; públicas, medios de comunicación; o estar basadas en experiencias anteriores de carácter similar (Colbert y Cuadrado, 2003; Berenguer y Cuadrado, 2003; Kotler y Scheff, 1997).

La implicación por el producto es, por otro lado, una de las variables más importantes pues está directamente relacionada con el riesgo que como espectadores asocian a su decisión de compra. Colbert y Cuadrado (2003: 100) señalan que el consumo cultural se encuentra estrechamente relacionado con el riesgo funcional. El riesgo funcional es la posibilidad de que un espectáculo o producto cultural no cubra las expectativas del consumidor y es común en sectores -como el cultural-en los que no es posible la prueba de producto antes de su compra (Colbert, 2007: 106). Sin embargo, un espectador puede reducir considerablemente este riesgo si busca la máxima cantidad de información posible sobre la obra en cuestión. Por ello, las recomendaciones de conocidos y amistades pueden ayudar a reducirlo. De hecho, Tomlinson y Roberts (2011: 288) han señalado que el boca a boca siempre ha sido una de las principales herramientas de referencia en el mundo de la cultura. Pero su influencia podría haber aumentado en el curso de los últimos años, por el impacto y progresiva presencia de las redes sociales y otras herramientas asociadas a las tecnologías de la información y la comunicación. Efectivamente, el creciente impacto de las redes sociales y de canales de comunicación como los blogs acrecientan el efecto potencial del boca a boca como herramienta promocional. De acuerdo con estos antecedentes y posibilidades, esta investigación se ha planteado como hipótesis que el boca a boca (re- 
comendaciones de amigos y conocidos) será la primera fuente de prescripción del consumo teatral y que serán los espectadores que asisten con menos frecuencia al teatro -por tanto, los que se ven más expuestos al riesgo funcional- los que se muestren más propensos a tener en cuenta los consejos y recomendaciones de sus círculos de amistades a la hora de asistir al teatro.

La influencia de las críticas, las reseñas o las entrevistas con el director o los actores ha sido escasamente estudiada en el ámbito del consumo teatral. A nivel internacional se han realizado estudios centrados en el impacto de la información cultural y las críticas publicadas en sectores como el cinematográfico. En un estudio de Basuroy, Chatterjee y Ravid (2003) se analizó la influencia de las críticas, tanto positivas como negativas, en la recaudación en taquilla. Los resultados revelaron que había una estrecha relación entre crítica y venta de entradas, sobre todo de las críticas negativas que reducían significativamente la venta de entradas. Estos resultados no fueron confirmados por estudios posteriores como el realizado por Wang (2005), en el que se demostró una mayor influencia de las críticas positivas. Con anterioridad, Eliashberg y Shugan (1997) habían concluido que los críticos cinematográficos influían indirectamente en el proceso de compra, pues aunque sus comentarios tenían efecto en los gustos de los espectadores no tenían un impacto directo en las taquillas, algo que también se apuntó en un estudio realizado por Austin (1983). Por su parte, el estudio realizado por Colbert y d'Astous (2003) sostiene que el grado de consulta de críticas depende de cuatro variables psicológicas: el grado de conocimiento cinematográfico, la autoestima del espectador, la sensibilidad a la presión social y el grado de implicación cultural. De esta manera, las diferentes variables se cruzan con el grado de asistencia y se concluye que aquellos que más acuden al cine muestran menor influencia a la críticas, mientras que los acuden menos serán más propensos a reforzar sus decisiones con la búsqueda de información y la consulta de críticas. El estudio, además, propone diferentes estrategias de promoción para aprovechar o disminuir la influencia de la crítica en cada caso.

En el marco de la críticas teatrales, Laplante y Lavoie (1986) realizaron un estudio de públicos en seis teatros de Montreal y analizaron, por una parte, la opinión de los espectadores sobre las críticas y, por otra, la relación entre el teatro, los medios de comunicación y los espectadores. Concluyeron que estos consultan con frecuencia las críticas teatrales, si bien, la mayoría estimaba que gran parte de las mismas se centraban en obras de teatro producidas por grandes compañías y daba escasa relevancia a los espectáculos de compañías jóvenes y de teatro experimental. El estudio señalaba, además, que el público más asiduo valoraba negativamente el escaso tratamiento otorgado a los elementos que componen la obra de teatro -el texto, la puesta en escena, la interpretación de los actores y la escenografía- en las críticas, frente al público esporádico que no prestaba atención a estos aspectos. Una segunda encuesta llevada a cabo por los mismos investigadores revelaba que la mitad de los espectadores entrevistados se informaron de la obra a través de los medios de comunicación -más concretamente de periódicos-, mientras que un tercio había acudido por recomendación de algún familiar o amigo. En lo que respecta a la críticas, un 20\% de los encuestados afirmaron consultarlas y un $90 \%$ reconocía que cuando leía una crítica favorable de 
una obra de teatro seguía la recomendación del crítico. Por su parte, el estudio realizado en Broadway por Reddy, Swaminathan y Motley (1998), señaló que los líderes de opinión, los expertos, los amigos y las reseñas publicadas en medios de comunicación eran fuentes fiables de información para el consumidor, sobre todo porque no se les atribuye un interés y por ello resultan persuasivos. Más recientemente, un estudio realizado en Londres (Senior, 2004) ha analizado el impacto de las críticas teatrales en términos económicos poniendo de manifiesto la influencia de estas -cuando tienen un carácter positivo- en el gasto teatral frente a otras alternativas. Así pues, los críticos, como líderes de opinión en el ámbito cultural, contribuyen al éxito o el fracaso de un producto artístico.

En España, la influencia de los diversos prescriptores en el consumo cultural ha sido un aspecto apenas estudiado. Un estudio sobre el consumo de artes escénicas publicado hace diez años (Luna, 2004: 19) señalaba que una parte de los espectadores considera que la verdadera causa del bajo porcentaje de asistencia al teatro radica en el elevado desconocimiento y la escasa respuesta del público local a la programación cultural. El estudio también analizaba la influencia de las críticas sobre los espectadores y mostraba que la recomendación de un amigo, un conocido o la confianza que provoca una buena crítica en los medios de comunicación provocan un estímulo externo añadido a la propensión personal hacia un determinado espectáculo cultural, ya que se trata de información externa que reduce el riesgo de asistir a una representación teatral. Una recomendación o una buena crítica aumentará la predisposición del público a asistir al teatro, sobre todo entre aquellos que suelen hacerlo con menos frecuencia.

Esa suposición se basa en el hecho de que, a menudo, la decisión de compra de un producto cultural se basa en una imitación o recomendación y el proceso de decisión -o parte de él-, se subordina a un tercer elemento, que debe ser creíble para el consumidor -lo que Colbert y Cuadrado (2003: 109) definen como un proceso subordinado-. La fuente de conformidad o imitación puede ser un amigo o un familiar, pero también un individuo considerado 'experto', algo muy propio de los productos culturales y artísticos. Cameron (1995: 322) menciona el análisis de Mosseto (1993) como uno de los pioneros en apuntar la tesis de que los críticos ayudan a los espectadores a apreciar el arte y a descifrar el trabajo artístico. Así, los críticos adquieren una importancia y responsabilidad que puede explicarse por la propia naturaleza de los productos culturales, más difíciles de juzgar y más arriesgados de consumir (Debenedetti, 2006: 44). Desde este punto de vista se suele asumir que "cuanto más intangible es el criterio por el que se valora un bien o servicio, más importantes resultan los prescriptores del gusto sobre el proceso de reconocimiento social y económico de este bien y servicio" (Ramon y Basso, 2010: 7). Kotler y Scheff (1997: 129) y Kolb (2005:109) también señalan la importancia de factores sociales como los grupos de referencia y los líderes de opinión que influyen en los espectadores. Estos factores sociales en ocasiones resultan decisivos porque pueden influir en la actitud y el comportamiento de los consumidores. Cuanto más valore una persona al líder de opinión, más influencia tendrá la opinión de este en la elección que haga esa persona, sobre todo en el caso de los intercambios de alta implicación donde el proceso de decisión de compra es mucho más complejo. Cuando el tipo de intercambio sea novedoso para la persona que rea- 
liza la elección o esta no sepa gran cosa sobre ese tipo de producto, se producirá una elevada presencia de este tipo de prescripción, sobre todo si la decisión de compra es considerada importante, cara o arriesgada (Kotler y Scheff, 1997; Sellas y Colomer, 2009). Es por ello que la investigación que se presenta plantea como hipótesis de trabajo que las críticas tendrán una mayor influencia tanto en el público que asiste con menos frecuencia al teatro como entre los espectadores de teatro comercial -frente a los espectadores de las salas alternativas o independientes-.

En definitiva y a modo de recapitulación, de acuerdo con la revisión de la literatura y de acuerdo con los hechos expuestos, la investigación que se presenta en este artículo pretende contrastar las siguientes cuatro hipótesis:

$\left(\mathrm{H}_{\mathrm{l}}\right.$ ) Las recomendaciones de amistades y conocidos (boca a boca) constituyen la más importante fuente de prescripción de la asistencia al teatro en la actualidad.

$\left(\mathrm{H}_{2}\right)$ Los espectadores que asisten con menos frecuencia al teatro se ven más influidos por las recomendaciones de sus amistades y conocidos (boca a boca) que aquellos que asisten más a menudo.

$\left(\mathrm{H}_{3}\right)$ Las críticas teatrales tienen una mayor influencia en el público que acude con menos frecuencia al teatro.

$\left(\mathrm{H}_{4}\right)$ Las críticas teatrales tienen una mayor influencia en los espectadores de teatro comercial -frente a los espectadores de las salas alternativas o independientes-.

\section{Método}

\subsection{Muestra}

La base empírica de esta investigación procede de una encuesta realizada a una muestra de 210 espectadores teatrales de la ciudad de Valencia. El trabajo de campo se llevó a cabo en tres salas teatrales de la ciudad: el Espacio Inestable, el Teatro Rialto y el Teatro Olympia. Estas tres salas fueron elegidas por su especialización en distintos géneros teatrales y por su orientación a diferentes perfiles de espectadores. No era intención de este estudio obtener una muestra representativa del conjunto de espectadores teatrales de la ciudad, sino obtener una muestra heterogénea que hiciera posible examinar el comportamiento de los espectadores teatrales en relación a la información procedente de los medios de comunicación independientemente de sus gustos teatrales específicos. El Espacio Inestable es una sala que acoge obras alternativas de teatro independiente y cuenta con un amplio número de seguidores. El Teatro Rialto es una sala pública que dirige su programación a un público más diverso y suele contar con artistas y autores teatrales de trayectoria destacada. Por último, el Teatro Olympia es una sala privada que acoge obras más accesibles y destinadas al gran público, apuesta por artistas presentes en el imaginario audiovisual colectivo y da cabida espectáculos familiares y de humor.

El número de encuestas se distribuyó proporcionalmente -se realizaron 70 encuestas en cada sala - con la finalidad de conseguir una representación adecuada del público de cada sala. Las entrevistas se realizaron en la antesala de cada uno de las tres salas teatrales y permitieron la observación indirecta de los comportamientos y hábitos culturales propios de los espectadores. Se aplicó un procedimiento de muestreo aleatorio con el fin de evitar entrevistar a aquellas personas que mostraban una mejor 
predisposición a la hora de participar en el estudio. El trabajo de campo se realizó entre el 18 de febrero y el 13 de mayo de 2012. Durante los casi tres meses en que tuvo lugar el trabajo de campo de esta investigación se realizaron diversas actuaciones teatrales en cada una de las tres salas, lo que aseguró la renovación del público asistente. En el Espacio Inestable se realizaron 15 representaciones de 4 obras diferentes, en el Teatro Rialto 13 de 2 y en el Teatro Olympia 10 de 3.

\subsection{Cuestionario}

El cuestionario aplicado en esta investigación fue elaborado de manera expresa para la misma. Los estudios sobre información cultural y críticas teatrales son escasos y no hay ninguno que incluya una aproximación empírica como la que se ha llevado a cabo en esta ocasión. Para su elaboración se tuvo en cuenta la investigación sobre la influencia y papel de la crítica en otros ámbitos culturales como el cine o la música, así también como el cuestionario de las últimas ediciones de la Encuesta de Hábitos Culturales en España (Ministerio de Cultura, 2011). Para facilitar la comunicación con las personas encuestadas, la aplicación del cuestionario fue acompañada de cartones que recogían las opciones de respuesta o escalas de aceptación y desagrado. Antes de la realización del trabajo de campo se llevó a cabo una prueba piloto con quince espectadores teatrales.

El cuestionario se estructuró en diferentes bloques. En el primer bloque se consideró fundamental contextualizar la información de la persona encuestada indagando en sus hábitos generales de consumo cultural para, seguidamente, recoger información sobre sus pautas de asistencia al teatro. A continuación se incluyó una serie de preguntas con las que se pretendía analizar los desencadenantes y aspectos que muestran una mayor influencia en la asistencia al teatro. Se incluyeron también variables sobre búsqueda de información teatral y el uso de Internet por parte de los espectadores teatrales. El siguiente bloque se centró en los factores de prescripción y en su grado de influencia y tras éste se incluyó un conjunto de preguntas de satisfacción con la programación y la información teatral. El cuestionario se cerraba con un bloque de preguntas de clasificación destinadas a conocer las características sociodemográficas de las personas entrevistadas.

\subsection{Análisis de datos}

La información contenida en los cuestionarios de las 210 entrevistas se volcó en una matriz de datos elaborada específicamente para este trabajo. Previamente fueron codificadas las respuestas de las preguntas abiertas. Tanto la matriz de datos como el conjunto de los análisis han sido realizados con el programa SPSS version 19.

Por lo que se refiere a las técnicas estadísticas aplicadas, para conocer el grado de significatividad de las variables relacionadas con la consulta de información previa a la obra teatral y la influencia de los factores de prescripción se realizaron diversos análisis Chi-cuadrado. Por otro lado, para identificar los factores que inciden en la decisión de asistir al teatro y para determinar la influencia de las reseñas, las críticas y los reportajes sobre esa asistencia se recurrió al análisis de varianza unidireccional (ANOVA oneway). En los casos en que éste resultaba estadísticamente significativo se aplicó el test de Tukey. En las comparaciones de dos grupos se recurrió a la T de Student. 


\section{Resultados}

La muestra entrevistada asiste una media de 16 veces al año a una representación teatral; 18,6 veces en el caso de los hombres y 14,4 las mujeres. Por rangos de edad, los espectadores mayores de 65 años son los que más asisten al teatro, con un promedio anual de 34,1, seguidos de las personas entre 20 y 35 años que acuden 15,7 veces, y las de 36 a 65 años que acuden una media de 14 veces al año. Los espectadores del Espacio Inestable son los que registran la tasa de asistencia más elevada: 27,9 veces al año, seguidos por los del Teatro Rialto con un promedio anual de 12,5 veces. Los del Teatro Olympia se reducen hasta una media anual de 7,7 veces.

En cuanto al interés por el teatro, en una escala de 1 a 10 el promedio global se sitúa en 8 , no existiendo diferencias significativas entre hombres y mujeres. Por grupos de edad, el mayor grado se registra entre los mayores de 65 años, con un promedio de 8,9 , seguidos por el grupo entre 50 y 65 años con una media de 8 . Por el contrario, la valoración más baja es la de los jóvenes entre 20 y 35 años que tan sólo se sitúan una décima por debajo del promedio global. Por nivel de estudios, se observa que las personas con estudios universitarios tienen más interés por el teatro que las que no lo son. Cuando se compara a los espectadores de las tres salas se detectan diferencias significativas: el interés medio por el teatro de los espectadores del Espacio Inestable es estadísticamente superior al de los espectadores de los Teatros Rialto y Olympia $(8,4$; 7,9 y 7,6 respectivamente). Por último, el interés de los espectadores aumenta proporcionalmente según el número de veces que asisten a una representación teatral. Los espectadores que acuden más de 10 veces registran un interés promedio de 8,9 frente a los que asisten de 4 a 10 veces cuya media es de 7,9. Por otro lado, los que asisten de 1 a 3 veces alcanzan un interés medio de 7 .

La tabla 1 (ver Anexo) recoge las respuestas relativas a las fuentes de información a las que habían recurrido los espectadores para informarse sobre la representación teatral a la que asistían. Aunque al tratarse de una pregunta de respuesta múltiple no se ha podido aplicar ninguna prueba de contraste estadístico, los resultados obtenidos no arrojan ninguna duda respecto a los círculos de conocidos o amistades como primera fuente de información: un 48,1\% de los entrevistados señaló que había tenido conocimiento de la obra de teatro que se representaba en ese momento a través de amigos o conocidos, un $18,1 \%$ por medio de la programación de la propia sala y un $16,2 \%$ a través de los medios de comunicación (críticas, reseñas, reportajes, entrevistas...). A continuación se sitúa la publicidad $(11,9 \%)$ y la información contenida en publicaciones de tipo cultural $(8,2)$, y ya con porcentajes mucho más reducidos la recomendación o consejo de algún profesional del sector $(2,4 \%)$. Estos resultados confirman la $H_{1}$ con la que se afirmaba que el boca a boca o recomendaciones de amigos y conocidos seguía constituyendo la instancia de prescripción más influyente de la asistencia al teatro en la actualidad. La hipótesis, además, se confirma en todos los grupos sociales incluidos en la investigación. Así pues, el boca a boca es la fuente de información más habitual, independientemente del sexo, la edad, el nivel de estudios, la frecuencia de asistencia y el tipo de teatro preferido.

Ahora bien, son los jóvenes entre 20 y 35 años los que tienen más en cuenta las opiniones de sus amigos y conocidos $(56,2 \%)$, porcentaje que desciende según aumenta 
la edad. La prensa es una fuente de consulta más utilizada por los espectadores de edad más avanzada, concretamente entre los mayores de 65 años, que la mencionan en un $23,1 \%$ de los casos. Este mismo segmento de edad se refiere a las publicaciones culturales y la propia programación de la sala (en un $23,1 \%$ y un $23,1 \%$ de los casos respectivamente). Los datos obtenidos ponen de manifiesto, por otro lado, que los espectadores que asisten con menos frecuencia al teatro son los que más se guían por las recomendaciones de amigos y conocidos $(58,8 \%)$. Por el contrario, los que asisten con más frecuencia (más de diez veces al año) son los que más recurren a los medios de comunicación $(18,2 \%)$, las publicaciones culturales $(10,4 \%)$ y la propia programación de las salas $(28,6 \%)$ para informarse. Se confirma así la $H_{2}$, según la cual, los espectadores que asisten con menos frecuencia al teatro se muestran más influidos por las recomendaciones de sus amistades que por los medios de comunicación. Este hecho está relacionado con el mayor grado de riesgo funcional al que se expone este tipo de espectadores y la consiguiente necesidad de reducirlo a través de información personal.

Los espectadores también exhiben pautas de comportamiento diferentes según el tipo de teatro del que son más habituales asistentes. Los espectadores del Teatro Olympia destacan por ser los que más se informan a través de la publicidad (24,3\%) y los medios de comunicación $(21,4 \%)$ y los que menos lo hacen a través de publicaciones de carácter cultural $(5,7 \%)$. En cuanto a los del Teatro Rialto, son los que más se informan por las recomendaciones de su círculo amistades $(58,6 \%)$ y los que menos recurren a los medios de comunicación $(11,4 \%)$ o a la propia programación de la sala $(12,9 \%)$. Por último, los espectadores del Espacio Inestable son los que mencionan en mayor medida las publicaciones culturales $(10 \%)$ y la difusión de la programación que realiza la propia sala $(27,1 \%)$.

Las variables relacionadas con los factores que podían incidir en la decisión de asistir al teatro se registraron en escalas de ' 0 ' a ' 10 ', en las que el ' 0 ' correspondía a la mínima importancia y el ' 10 ' al máximo. Para identificar la existencia de diferencias estadísticamente significativas se ha aplicado la T de Student (sexo y nivel de estudios) y el Análisis de Varianza unidireccional (edad, frecuencia de asistencia, tipo de sala teatral), en algunos casos complementado con el Test de Tukey. Los resultados se presentan en la tabla 2 (ver Anexo).

Por lo que se refiere a la variable sexo, se ha comprobado que las mujeres dan más importancia que los hombres al hecho de ver la obra anunciada en prensa o televisión, también a que tenga buenas críticas y a que sea famosa y se hable mucho de ella. En cuanto al nivel de estudios, es significativa la influencia que tiene para las personas con estudios universitarios que el director de la obra sea conocido frente a la poca importancia con la que valoran esta variable los encuestados sin formación universitaria. Por otro lado, los espectadores más jóvenes (grupos de 20 a 35 años y de 36 a 49 años) otorgan una mayor influencia a las opiniones de sus familiares y amistades que los espectadores con más de 65 años. La frecuencia de asistencia al teatro es también una variable asociada de manera significativa a diversos factores de prescripción. Los espectadores más asiduos resultan ser los que menos se dejan influir por haber visto anunciada una obra en la prensa o la televisión, por las buenas críticas o 
por el hecho de que tenga notoriedad y se hable mucho de ella. Frente a ellos, los espectadores esporádicos revelan una mayor influencia de todas estas instancias de prescripción. Se contrasta de este modo la $H_{3}$, según la cual, las críticas teatrales tienen un mayor impacto en el público que asiste con menos frecuencia al teatro.

Otro resultado estadísticamente significativo tiene que ver con el tipo de sala al que suele acudir el espectador teatral. El estudio revela que los espectadores de teatro comercial (representados por el público del Teatro Olympia) conceden más importancia a la notoriedad de los actores y menos al director de la obra. Se encuentran también diferencias significativas en lo que se refiere a la influencia de los medios de comunicación. Los espectadores del Teatro Olympia se muestran más proclives a tener en cuenta las críticas teatrales y la publicidad en el momento de tomar la decisión de asistir al teatro. Por su parte, el público del Espacio Inestable es el que menos valora estos factores y, por tanto, el que menos influencias recibe de estas instancias de prescripción. Se corrobora, así, la hipótesis $H_{4}$, según la cual las críticas influyen más en los espectadores de teatro comercial que en los de salas alternativas o independientes.

Al profundizar en el papel de los medios de comunicación como fuentes de información cultural mediante variables adicionales, los resultados han revelado que una gran parte de los espectadores de teatro habían llevado a cabo una consulta de información previa a la representación de la obra teatral. Más concretamente, la tabla 3 (ver Anexo) muestra que un 54,3\% de los encuestados había leído una reseña antes de asistir al teatro, proporción que se reducía hasta un $19 \%$ en el caso de los reportajes. Además, un 16,2\% había leído una crítica y un 2,4\% una entrevista a los actores o al director. En la tabla 3 (en Anexo)se encuentra un análisis pormenorizado del perfil de espectador que consulta las críticas teatrales antes de asistir a una representación. Los datos obtenidos ponen de manifiesto que las críticas teatrales son más consultadas por las personas entre 50 y 65 años (lo hacen en un $20 \%$ de los casos), y casi con la misma proporción se sitúan los jóvenes con edades comprendidas entre los 20 y los 35 años $(19,1 \%)$. Por otro lado, los que asisten con menor frecuencia al teatro son los que más consultan las críticas teatrales $(23,5 \%)$ frente aquellos que van más de diez veces al año que son quienes menos las consultan $(9,1 \%)$. Además, los espectadores del Teatro Olympia son los que más consultan las críticas sobre una obra $(21,4 \%)$, seguidos de los espectadores del Teatro Rialto (20\%) y con un porcentaje bastante menor, el Espacio Inestable $(7,1 \%)$. El test de Chi-cuadrado revela la existencia de una relación estadística significativa con esta última variable. En este sentido, la información contenida en la tabla 3 ofrece apoyo empírico adicional a las hipótesis $H_{3}$ y $H_{4}$-ya contrastadas a partir de los datos contenidos en la tabla 2 (en Anexo)-, pues la proporción de espectadores de teatro comercial (público del Teatro Olympia) que consulta las críticas teatrales es significativamente superior a la proporción registrada entre los espectadores de otras salas teatrales. Así mismo, el análisis de residuos tipificados corregidos revela que los espectadores del Espacio Inestable consultan con mucha menor asiduidad las críticas teatrales que el resto de públicos. Por tanto, teniendo en cuenta estos datos, quedarían de nuevo confirmadas las hipótesis $\mathrm{H}_{3}$ y $\mathrm{H}_{4}$ según las cuales las críticas teatrales influyen más en los espectadores que menos asisten al teatro y en los espectadores de teatro comercial -frente a los espectadores más 
asiduos y el público de las salas independientes, respectivamente-. Dicho de otro modo, los resultados obtenidos revalidan la tesis de que los espectadores de salas teatrales con una orientación más comercial se muestran más proclives a tener en cuenta los elementos de prescripción presentes en los medios de comunicación que aquellos espectadores de salas con programaciones de carácter alternativo o independiente.

La tabla 4 (ver Anexo) muestra información sobre el grado en que las reseñas, críticas y reportajes teatrales tienen efecto en la decisión de asistir al teatro. Se observa, en primer lugar, que su efecto es mayor en las mujeres que en los hombres: les ayudan más a decidirse y les influyen más. Estos datos corroboran los resultados ya examinados de la tabla 2 (en Anexo) y revelan que existe una relación significativa entre la variable sexo y el papel de este tipo de prescriptores. En segundo lugar, la aplicación de la T de Student también revela que el nivel de estudios arroja diferencias significativas ya que los espectadores con estudios universitarios consultan más y están más influidos por las reseñas, críticas y reportajes que aquellos cuyo nivel de estudios es inferior. Por otro lado, el análisis de varianza y el test de Tukey ofrecen resultados estadísticamente significativos en lo que se refiere a la frecuencia de asistencia al teatro: los espectadores que asisten al teatro con menos frecuencia se muestran más influidos por las críticas teatrales que aquellos que lo hacen más a menudo (más de diez veces al año). Estos datos aportan mayor consistencia a la verificación de la $H_{3}$-ya confirmada a propósito de las tablas 2 y 3 (en Anexo)- según la cual las críticas influyen más en el público que asiste con menos frecuencia al teatro.

Una última variable -referida a la influencia de una mala reseña, crítica o reportaje- permite abundar en los resultados ya expuestos. Aunque no se han detectado diferencias significativas en virtud del sexo, la edad y el nivel de estudios, el test de Chi-cuadrado y el análisis de residuos tipificados revelan que los espectadores del Espacio Inestable son los que menos se dejan influir por una mala reseña, crítica o reportaje frente a los espectadores del Teatro Rialto y el Teatro Olympia, mucho más proclives a tener en cuenta estas prescripciones de carácter negativo. Estos datos permiten confirmar, como ya se había apuntado al examinar las tablas 2, 3 y 4 (en Anexo), la $H_{3}$ y la $H_{4}$ que sostienen que las críticas -en este caso, las malas críticas-influyen más en los espectadores de teatro comercial y en los que menos asisten al teatro frente a los espectadores de las salas independientes.

\section{Conclusiones}

La investigación presentada en este artículo ha profundizado en los aspectos que inciden en la decisión de asistir a una representación teatral así como el grado en que lo hacen. El riesgo funcional propio de las decisiones de compra en sectores como el cultural hace pensar en la necesidad de información así como en la posible influencia de diversas instancias de prescripción a la hora de tomar la decisión de asistir al teatro. Partiendo de ese planteamiento y de acuerdo con la revisión de la escasa literatura científica sobre el tema existente, este estudio se proponía contrastar cuatro hipótesis relativas al papel de la comunicación boca a boca, los medios de comunicación y las críticas teatrales en la asistencia al teatro, así como al perfil de los grupos sociales en que la presencia de estas instancias de prescripción sería más acentuada. Para abordar 
estos objetivos se diseñó una encuesta $a d$ hoc en la que se entrevistó a 210 espectadores de tres salas teatrales de la ciudad de Valencia.

El estudio ha puesto de manifiesto que en torno a la mitad de los espectadores teatrales suelen buscar información antes de asistir a una representación teatral, sobre todo los espectadores de mayor edad y los que lo hacen con más frecuencia. La búsqueda de información reduce el riesgo que supone asistir a la representación de una nueva obra de teatro. Un riesgo común en el sector de los servicios donde el producto no puede ser probado antes de la compra (Colbert y Cuadrado, 2003: 100). Para asegurar una compra satisfactoria, el público trata de obtener información sobre la obra antes de asistir a su representación. Aunque los motivos de esta esta búsqueda de información pueden ser diversos a menudo tienen que ver con experiencias anteriores insatisfactorias (Kotler y Scheff, 1997: 156) o con el desconocimiento y la dificultad que supone juzgar un espectáculo teatral (Sellas y Colomer, 2009: 21). El estudio también ha evidenciado que los espectadores que asisten con menos frecuencia al teatro se ven más influidos por el boca a boca y las recomendaciones de los amigos que por los medios de comunicación. Por el contrario, aquellos que asisten con más frecuencia (más de 10 veces al año) son los que más recurren a informarse a través de los medios de comunicación, las publicaciones culturales y la propia programación de las salas.

En lo relativo a los factores de prescripción, se ha observado que los más utilizados por los espectadores son las reseñas, los reportajes, las críticas y, por último, las entrevistas al director o a los actores del reparto. Al profundizar en el objeto específico de esta estudio, las críticas, se ha podido comprobar que el tipo de espectador que más las consultan y se dejan influenciar por ellas es una mujer, entre 50 y 65 años, sin estudios universitarios y que acude entre tres o menos veces al teatro al cabo de un año; un perfil que responde en gran medida al espectador de teatro comercial que caracteriza al público del Teatro Olympia. La conclusión más evidente a este respecto es que los espectadores teatrales asumen el riesgo inherente al consumo teatral, sobre todo por la incertidumbre previa que les proporciona la experiencia estética que van a disfrutar. Es por ello que una parte de ese público trata de paliar ese riesgo buscando información y consultando críticas teatrales. Hay, por tanto, una relación estrecha entre el producto escénico y la información que ofrecen los medios de comunicación como reductores de ese riesgo.

Queda para investigaciones futuras el análisis de las instancias de prescripción y su influencia a la hora de propiciar la asistencia a una representación teatral a través de estudios cualitativos que permitan conocer tanto las inquietudes de los espectadores, como la perspectiva de los artistas y los creadores. Este estudio también ha pretendido la apertura de nuevas líneas de investigación en las que trabajar para mejorar el conocimiento de los hábitos y prácticas de consumo cultural. En este sentido, es esencial delimitar el impacto diferencial de los distintos tipos de críticas y piezas de información teatral, así como del medio de comunicación en que se publican, sin olvidar el papel de Internet o las críticas informales, cada vez más presentes en las redes sociales y en los blogs y, por tanto, con grandes posibilidades de influir en el consumo cultural. 


\section{Referencias bibliográficas}

AA.VV (2011): Plan General del Teatro (revisión 2011), Ministerio de Cultura - Gobierno de España. http://www.mcu.es/artesEscenicas/docs/Plan_Generaldel_Teatro _rev2011.pdf

AUSTIN, Bruce (1983): "Critics and consumers evaluations of motion pictures. A longitudinal test of the taste culture and elitist hypotheses". Journal of Popular Film and Television, Vol. 10 (4), pp. 156-167. http://dx.doi.org/10.1080/01956051 .1983 .10661938

BASUROY, Suman; CHATTERJEE, Subimal; y RAVID, S. Abraham (2003): "How critical are critical reviews? The box office effects of film critics, star power, and budgets". Journal of Marketing, Vol. 67 (4), pp. 103-117. http://dx.doi.org/10.1509 /jmkg.67.4.103.18692

BERENGUER CONTRÍ, Gloria y CUADRADO GARCÍA, Manuel (2003): "El comportamiento del consumidor de actividades en vivo". Experiencias y técnicas en la gestión del ocio, pp. 161-168. Universidad de Deusto; Deustuko Unibertsitatea.

CAMERON, Samuel (1995): "On the role of critics in the culture industry". Journal of Cultural Economics, Vol. 19 (4), pp. 321-331. http://dx.doi.org/10.1007 /BF01073994

COLBERT, François y D'ASTOUS, Alain (2003): "La consultation de critiques de films et son impact sur la consommation". Gestion, Vol. 28 (1), pp. 12.

COLBERT, François y CUADRADO GARCÍA, Manuel (2003): Marketing de las artes y la cultura. Barcelona, Ariel.

COLBERT, François (2007): Le marketing des arts et de la culture. Montréal, Gaë$\tan$ Morin.

COLOMER, Jaume y GARRIDO, Andreu (2010): "Los públicos de las artes escénicas". Escenium, Foro internacional de las artes escénicas. Bilbao, 10-12 de febrero de 2010.

CUADRADO GARCÍA, Manuel y BERENGUER CONTRÍ, Gloria (2002): El consumo de servicios culturales. Escuela Superior de Gestión Comercial y Marketing, ESIC.

DEBENEDETTI, Stéphane (2006): "L'impact de la critique de presse sur la consommation culturelle: Un essai de synthèse dans le champ cinématographique". Recherche et Applications en Marketing, Vol. 21 (2), pp. 43-59,106.

ELIASHBERG, Jehoshua y SHUGAN, Steven (1997): "Film critics: Influencers or predictors?", The Journal of Marketing, Vol. 61 (2), pp. 68-78. http://dx.doi.org/10.2307/1251831

ENGEL, James F.; BLACKWELL, Roger D.; and MINIARD, Paul W. (1993): Consumer behaviour. Fort Worth, Texas, Dryden Press.

FERNÁNDEZ TORRES, Alberto (1995): "Notas para una posible reflexión sobre posibles opciones de la crítica teatral". ADE teatro: Revista de la Asociación de Directores de Escena de España, (43-44), pp. 51-57. 
IZQUIERDO EXPÓSITO, Violeta (2014): “Análisis de la información artística en los medios escritos generalistas españoles (El País, El Mundo y ABC)". Estudios sobre el Mensaje Periodístico, Vol. 20 (2), pp. 1121-1130. Madrid, Servicio de Publicaciones de la Universidad Complutense.

KOLB, Bonita M. (2005): Marketing for cultural organisations: new strategies for attracting audiences to classical music, dance, museums, theatre and opera. London, Thomson Learning.

KOTLER, Philip y SCHEFF, Joanne (1997): Marketing de las artes escénicas. Madrid, Fundación Autor.

LAPLANTE, Benoît et LAVOIE, Pierre (1986): "La critique et son public: Enquêtes", Jeu: revue de théâtre, vol. 40, pp. 94-110.

LORENTE BILBAO, José Ignacio (2013): "Comunicación crítica e investigación en artes escénicas”. Estudios sobre el Mensaje Periodístico, Vol. 19 (2), pp. 10291045. Madrid, Servicio de Publicaciones de la Universidad Complutense.

LUNA AROCAS, Roberto (2004): El consumo de teatro y danza en la ciudad de Valencia: un analisis desde la dirección estratégica. Valencia, Promolibro.

MOSSETTO, Gianfranco (1993): Aesthetics and economics. Dordrecht, Kluwer Academic Publishers.

RAMON, Gerard y BASSO, Ana Luisa (2010): Hacia un mapa de públicos de las artes escénicas a partir de principales estudios sobre públicos culturales a nivel internacional. Escenium, Foro internacional de las artes escénicas. Bilbao, 10-12 de febrero de 2010.

REDDY, Srinivas K.; SWAMINATHAN, Vanitha; and MOTLEY, Carol M. (1998): "Exploring the determinants of Broadway show success". Journal of Marketing Research, Vol. 35 (3), pp. 370-383. http://dx.doi.org/10.2307/3152034

SÁNCHEZ DE HORCAJO, Juan José (1999): Los teatros madrileños: un estudio sociológico. Madrid, Ediciones Libertarias.

SELLAS, Jordi y COLOMER, Jaume (2009): Marketing de las artes escénicas: creación y desarrollo de públicos. Barcelona, Bissap Consulting.

SENIOR, Ian (2004): “Theatre critics and theatregoing”, Economic Affairs, Vol. 24 (2), pp. 65-69. http://dx.doi.org/10.1111/j.1468-0270.2004.00478.x

TOMLINSON, Roger y ROBERTS, Tim (2011): Aforo completo: cómo convertir los datos en audiencias. Madrid, Ediciones Autor.

WANG, Alex (2005): "The effects of expert and consumer endorsements on audience response", Journal of Advertising Research, Vol. 45 (4), pp. 402-412. http://dx.doi.org/10.1017/S0021849905050452

María Teresa Logroño Tormo es comunicadora, periodista y gestora cultural e investigadora en la Facultad de Ciencias Sociales de la Universitat de València. 


\section{Anexo. Tablas de resultados}

\begin{tabular}{|c|c|c|c|c|c|c|c|c|c|c|c|c|c|c|c|}
\hline$\stackrel{\text { on }}{0}$ & $\stackrel{\text { ํ }}{\stackrel{2}{n}}$ & $\stackrel{\stackrel{\circ}{r}}{\stackrel{+}{+}}$ & \begin{tabular}{l} 
iे \\
in \\
\multirow{7}{*}{}
\end{tabular} & $\begin{array}{l}\text { ठ̊. } \\
\text { के }\end{array}$ & $\begin{array}{l}\text { ठें } \\
0\end{array}$ & $\stackrel{\stackrel{\circ}{n}}{\stackrel{2}{n}}$ & $\begin{array}{l}\text { ते } \\
\text { ํ}\end{array}$ & ㅇํㅇ & $\begin{array}{l}\text { مे } \\
\text { in }\end{array}$ & $\begin{array}{l}\text { ते } \\
\text { הे }\end{array}$ & $\begin{array}{l}\stackrel{0}{~} \\
\text { in }\end{array}$ & $\begin{array}{l}\stackrel{0}{ } \\
\stackrel{m}{*}\end{array}$ & $\begin{array}{l}\stackrel{0}{n^{\circ}} \\
\text { in }\end{array}$ & $\frac{\partial}{\pi}$ & $\begin{array}{l}\stackrel{0}{i} \\
\text { in }\end{array}$ \\
\hline 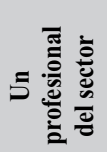 & $\frac{\stackrel{\circ}{r}}{i}$ & $\stackrel{\vec{b}}{b_{-}^{0}}$ & $\stackrel{\stackrel{\circ}{+}}{\stackrel{\text { fi }}{2}}$ & $\stackrel{\circ}{\stackrel{\circ}{=}}$ & $\begin{array}{l}\text { ठें } \\
\text { i }\end{array}$ & 1 & $\frac{\stackrel{0}{0}^{\circ}}{\vec{i}}$ & $\begin{array}{l}\text { in } \\
\text { in }\end{array}$ & i & I & 总 & $\frac{0}{\stackrel{0}{n}}$ & $\stackrel{\stackrel{\circ}{+}}{\stackrel{\circ}{\sim}}$ & i & $\stackrel{\stackrel{\circ}{+}}{\stackrel{0}{+}}$ \\
\hline 苞 & $\begin{array}{l}\stackrel{0}{ } \\
\infty \\
\text { مे }\end{array}$ & $\stackrel{\circ}{\circ}$ & ふूे & $\begin{array}{l}\dot{b}_{\infty}^{\circ} \\
\infty^{\circ}\end{array}$ & $\begin{array}{l}\stackrel{0}{\circ} \\
\dot{f}\end{array}$ & $\frac{\partial}{\partial}$ & 离 & $\begin{array}{l}\stackrel{0}{0} \\
\text { के }\end{array}$ & $\stackrel{\stackrel{\circ}{+}}{\stackrel{+}{+}}$ & ڤे & 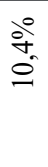 & $\begin{array}{l}\text { 号 } \\
\text { O }\end{array}$ & $\begin{array}{l}\dot{b}_{0}^{\circ} \\
\infty^{\circ}\end{array}$ & $\begin{array}{l}\stackrel{0}{\circ} \\
\text { in }\end{array}$ & $\frac{\partial^{0}}{\infty}$ \\
\hline 丞 & $\stackrel{\circ}{\Xi}$ & i̊ & ふ̆ & $\begin{array}{l}\text { वें } \\
\text { อे } \\
0\end{array}$ & $\begin{array}{l}\text { 今े } \\
\text { ¿े }\end{array}$ & i & $\stackrel{े}{0}_{\infty}^{\circ}$ & $\begin{array}{l}\dot{0} \\
\stackrel{2}{n}\end{array}$ & $\stackrel{\circ}{\stackrel{\infty}{=}}$ & $\begin{array}{l}\text { बें } \\
\text { فे }\end{array}$ & $\stackrel{\circ}{\infty}$ & i & 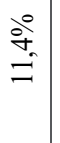 & $\begin{array}{l}\stackrel{0}{\circ} \\
\stackrel{\sim}{\sim}\end{array}$ & $\stackrel{\circ}{=}$ \\
\hline 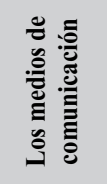 & $\begin{array}{l}\text { ठें } \\
\dot{ \pm}\end{array}$ & $\stackrel{\stackrel{0}{\beth}}{\text { ㄱ }}$ & $\begin{array}{c}\stackrel{0}{i} \\
\stackrel{2}{n}\end{array}$ & ํํㄹ & $\begin{array}{l}\text { ठ̊ } \\
\text { ¿े }\end{array}$ & $\frac{\partial^{\circ}}{\partial}$ & $\begin{array}{l}\text { dें } \\
\text { İ }\end{array}$ & $\begin{array}{l}\stackrel{0}{0} \\
\infty \\
0\end{array}$ & خें & $\begin{array}{l}\stackrel{0}{\infty} \\
\stackrel{一}{-}\end{array}$ & $\begin{array}{l}\stackrel{0}{0} \\
\infty\end{array}$ & $\frac{{ }^{\circ}}{n^{\circ}}$ & 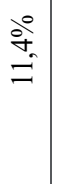 & $\frac{\stackrel{\circ}{+}}{\vec{v}}$ & तें \\
\hline 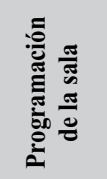 & $\begin{array}{l}\dot{o}^{\circ} \\
\text { ì }\end{array}$ & 迥 & $\begin{array}{l}\text { bे } \\
\text { İ }\end{array}$ & $\frac{\stackrel{0}{i}}{\stackrel{i}{i}}$ & $\begin{array}{l}\text { 8ें } \\
\text { हें }\end{array}$ & $\frac{\partial}{\partial}$ & $\begin{array}{l}\stackrel{0}{0} \\
\infty \\
\dot{\text { ¿े }}\end{array}$ & 导 & 导 & 亏ूे & $\begin{array}{l}\dot{b}_{0}^{0} \\
\infty_{i}^{\circ}\end{array}$ & $\frac{0}{\stackrel{\Delta}{\Delta}}$ & $\begin{array}{l}\text { aे } \\
\text { ปे }\end{array}$ & $\begin{array}{l}\stackrel{\circ}{\grave{~}} \\
\stackrel{ \pm}{ \pm}\end{array}$ & $\frac{\stackrel{0}{\infty}}{\infty^{\circ}}$ \\
\hline 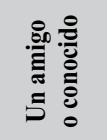 & $\begin{array}{l}\text { ळे } \\
\text { ซे }\end{array}$ & $\begin{array}{l}\stackrel{0}{0} \\
\infty \\
\dot{n}\end{array}$ & $\begin{array}{l}\text { तें } \\
\text { हैं }\end{array}$ & $\begin{array}{l}\dot{b}^{0} \\
\text { oे }\end{array}$ & $\begin{array}{l}\text { 8े } \\
\text { \&ें }\end{array}$ & $\begin{array}{l}\stackrel{0}{\circ} \\
\infty \\
\infty\end{array}$ & $\begin{array}{l}\stackrel{0}{0} \\
\infty \\
i\end{array}$ & $\frac{\partial}{a}$ & $\begin{array}{l}\infty^{\circ} \\
\infty \\
\infty \\
\infty\end{array}$ & 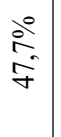 & $\begin{array}{l}\hat{o}^{\circ} \\
\text { ळे }\end{array}$ & $\begin{array}{l}\dot{b}^{\circ} \\
\text { o } \\
\dot{c}\end{array}$ & $\begin{array}{l}\dot{b}^{\circ} \\
\infty^{\circ} \\
i\end{array}$ & $\frac{\partial}{n}$ & $\frac{\partial^{\circ}}{\infty}$ \\
\hline \multirow{2}{*}{ 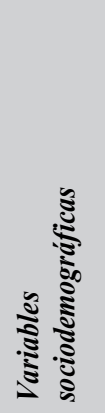 } & 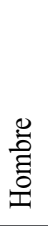 & $\cdot \frac{\dot{\vec{E}}}{\vec{\Xi}}$ & 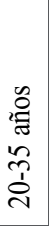 & 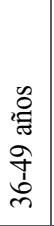 & 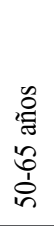 & 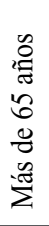 & 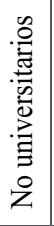 & 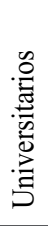 & 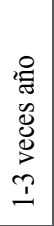 & 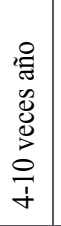 & 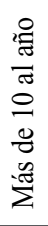 & 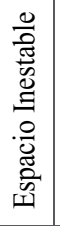 & 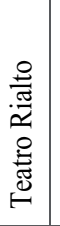 & 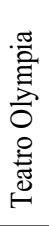 & \\
\hline & \multicolumn{2}{|c|}{ 矛 } & \multicolumn{4}{|c|}{ 莺 } & \multicolumn{2}{|c|}{ 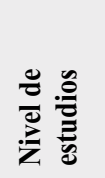 } & \multicolumn{3}{|c|}{ 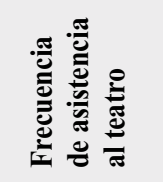 } & \multicolumn{3}{|c|}{ 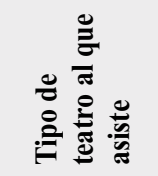 } & 苞 \\
\hline
\end{tabular}




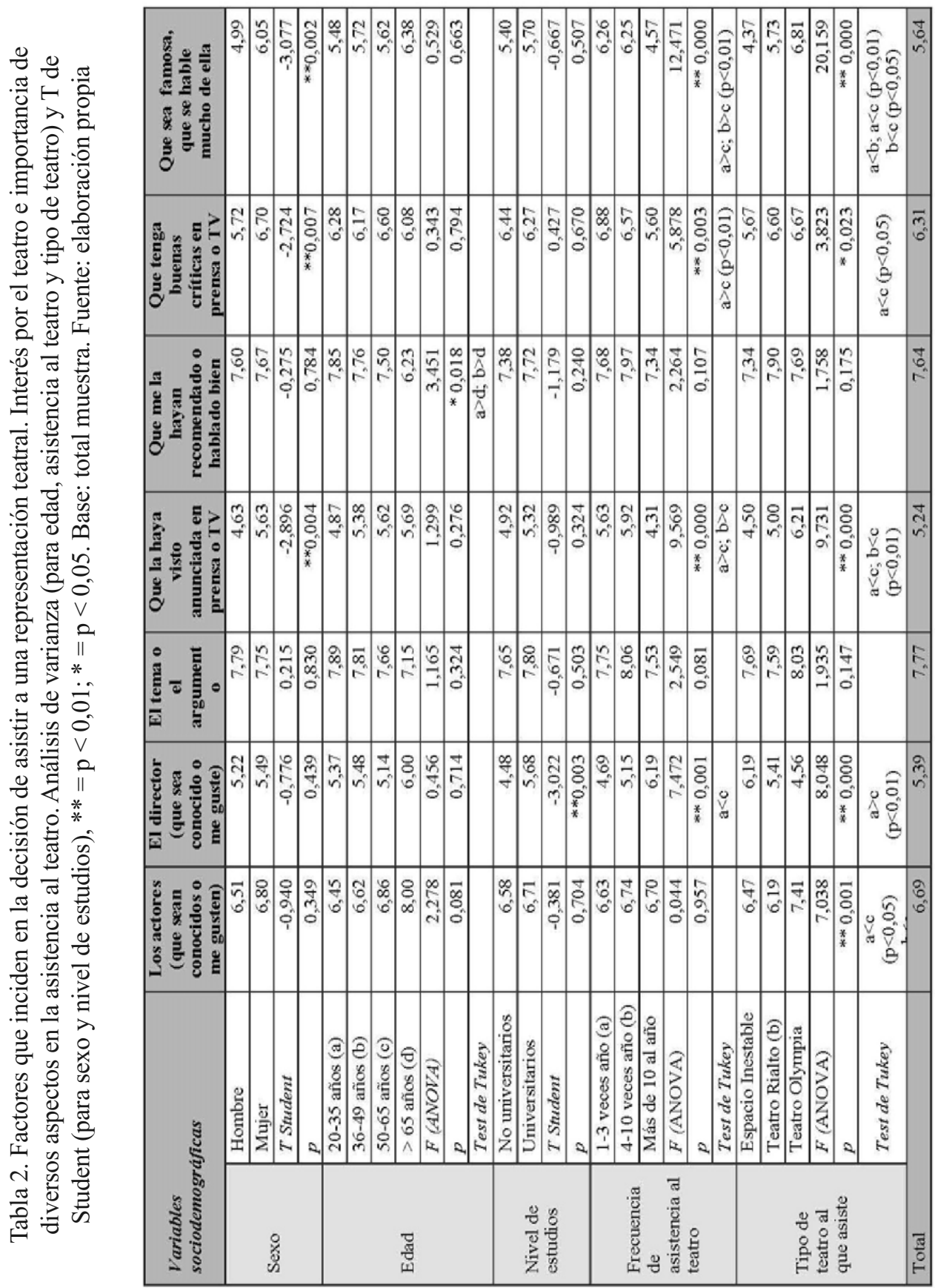




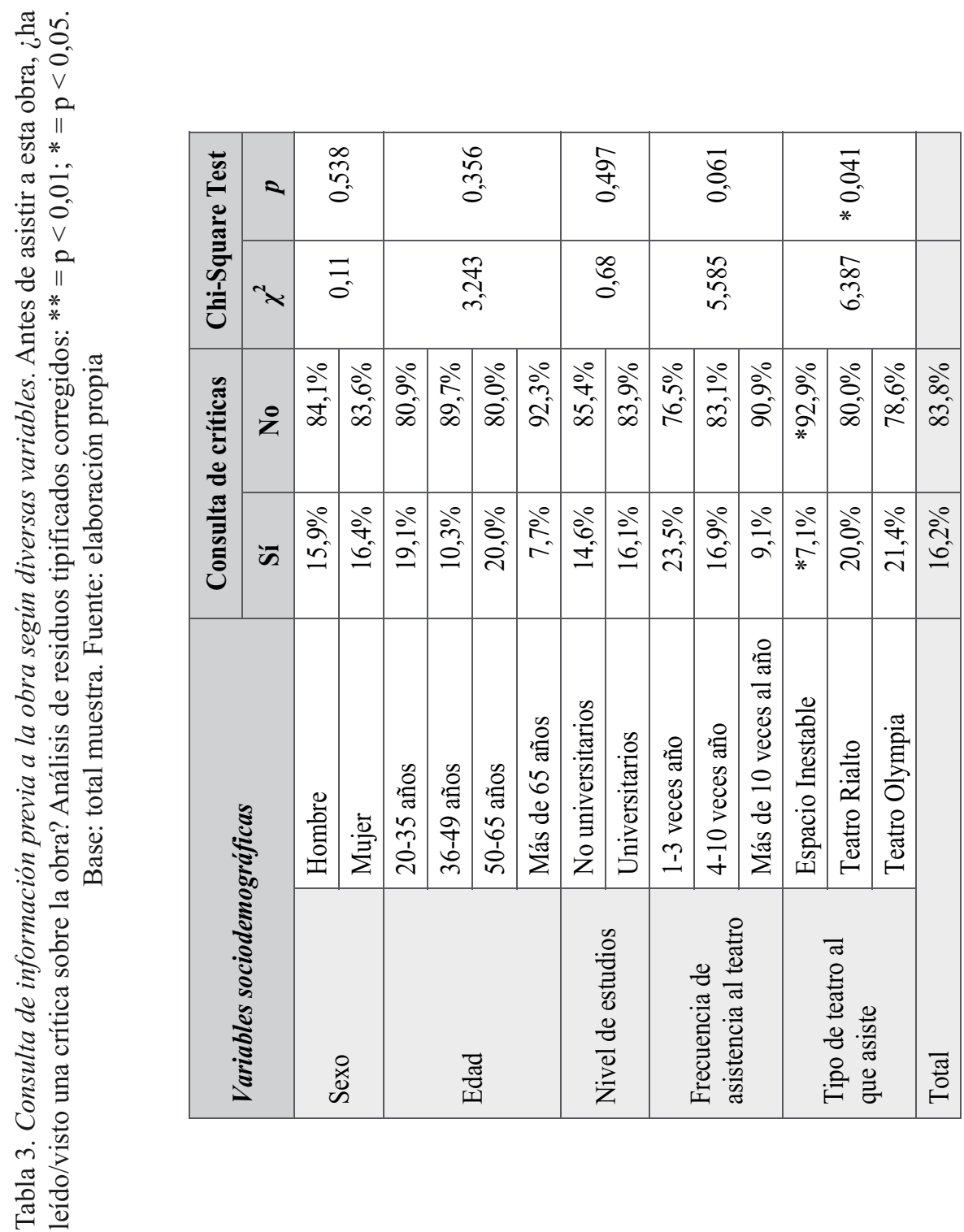




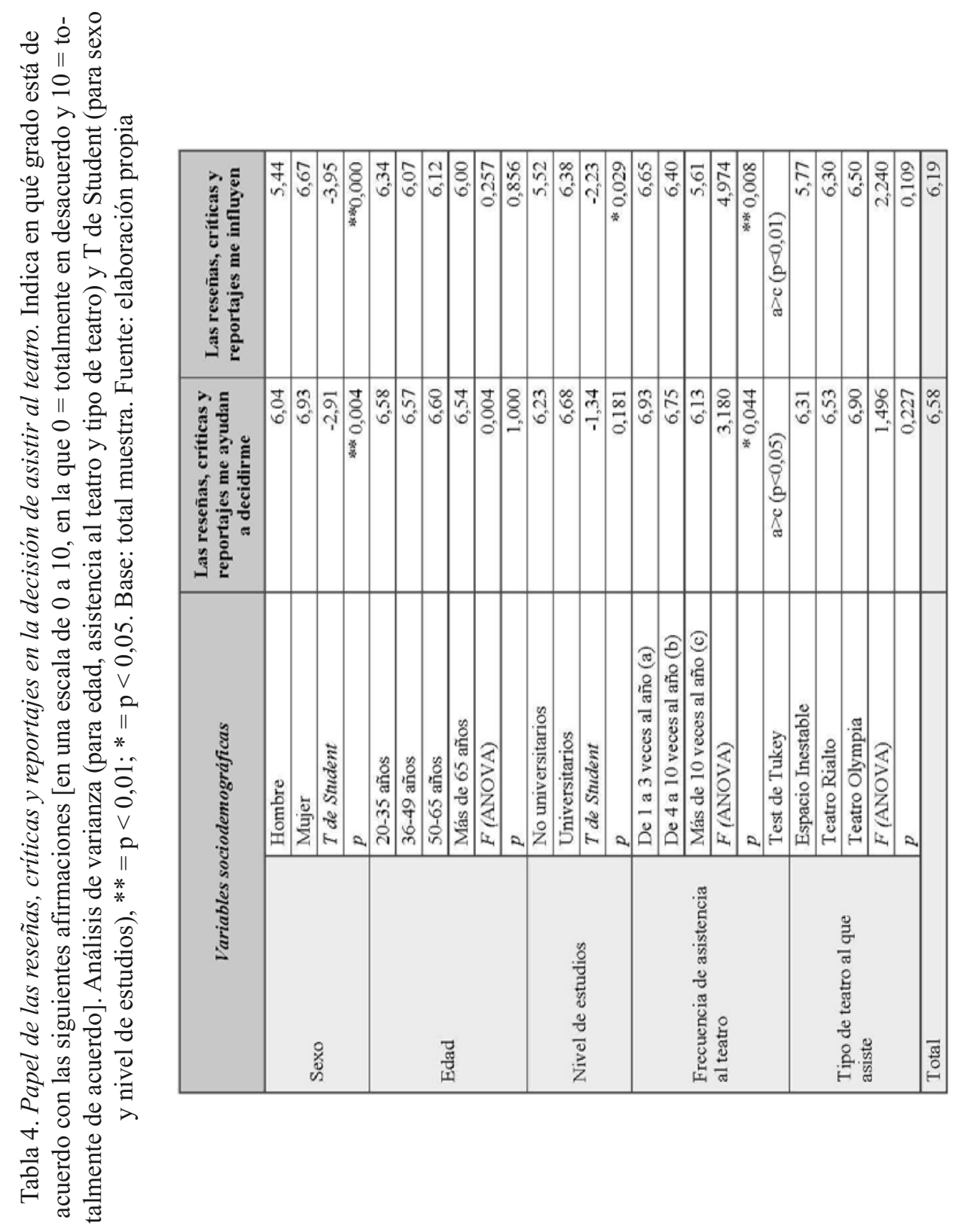




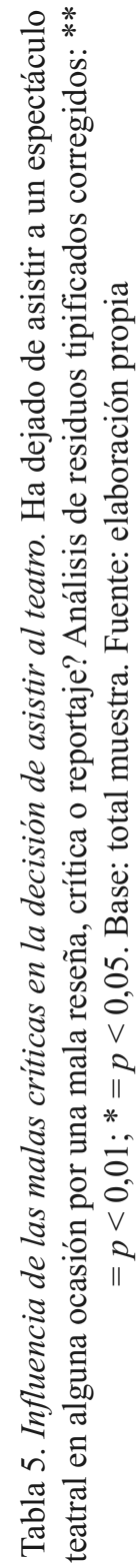

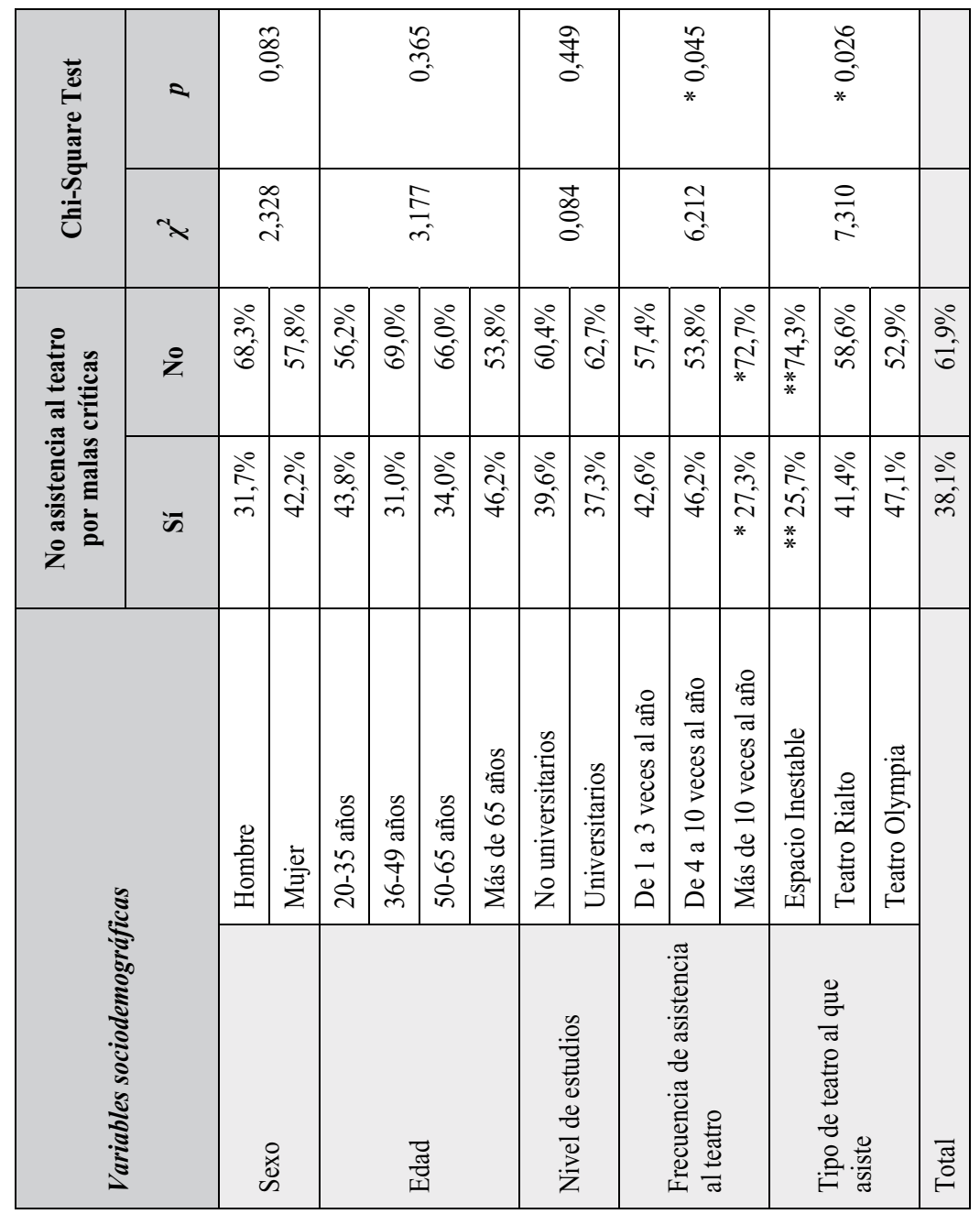

